\title{
Development of a New Approach for the Therapy of Prostate Cancer with SPOP Mutations
}

\author{
David Lu, Jason J. Lee, Allen J. Lee, Ray M. Lee* \\ Celestra Life Science LLC., Richmond, USA \\ Email: ${ }^{*}$ richvain@gmail.com
}

Received 7 July 2015; accepted 1 September 2015; published 4 September 2015

Copyright (C) 2015 by authors and Scientific Research Publishing Inc.

This work is licensed under the Creative Commons Attribution International License (CC BY). http://creativecommons.org/licenses/by/4.0/

(c) (i) Open Access

\begin{abstract}
Advanced prostate cancer is treated with androgen deprivation, but most patients eventually progress and need new therapy. Recent genomic/exomic sequencing identified SPOP as the most frequently mutated gene in $6 \%-15 \%$ of prostate cancer. Based on the function of SPOP as a ubiquitin ligase in protein degradation, it was hypothesized that loss-of-function mutations of SPOP led to accumulation of SPOP substrates that enhance androgen receptor activity and facilitate prostate cancer formation. SPOP substrates could thus be potential targets for treatment of androgen-sensitive prostate cancer. PubMed and BLAST search identified that Gli, SRC-3, and AWP1 are SPOP substrates, and that inhibition of PRK-1, a binding partner of AWP1, by lestaurtinib suppressed androgen receptor activity. LNCaP, PC3, DU145 and 22RV1 prostate cancer cells were used to evaluate the effect of lestaurtinib. LNCaP cells, an androgen-sensitive prostate cancer cell line, were the most sensitive. SRC-3 protein decreased when LNCaP cells were treated with lestaurtinib; whereas PRK-1 increased in nucleus after lestaurtinib treatment. These data suggest that lestaurtinib modulates SRC-3 and PRK-1 to induced cell death in androgen-sensitive prostate cancer, and could be a useful agent for future development for prostate cancer with SPOP mutations.
\end{abstract}

\section{Keywords}

Prostate Cancer, SPOP, SRC-3, PRK-1, Lestaurtinib

\section{Introduction}

Prostate cancer is one of the most common cancers in men worldwide. In 2012, it was estimated that there were

${ }^{*}$ Corresponding author. 
241,740 new cases and 28,170 deaths due to prostate cancer in the US alone. Prostate cancer is a cancer that depends on androgen to grow; hence hormonal therapy with androgen ablation is the mainstream treatment of choice for advanced or metastatic prostate cancer. It is an effective treatment and can keep disease under control for years. However, patients eventually fail and develop androgen refractory disease. There is a high unmet medical need for new treatments. Using next generation sequencing, Barbieri et al. identified several genes mutated in prostate cancer with SPOP as the most frequent one in $6 \%-15 \%$ of samples they examined [1]. SPOP is a Cullin3-based E3 ubiquitin ligase that adds ubiquitin to target proteins to facilitate degradation [2]. Interestingly, all the mutations of SPOP detected in prostate cancer are within the substrate-binding pocket of SPOP [1], indicating that mutations would affect the substrate-binding ability to alter or even abolish the activity of SPOP as E3 ubiquitin ligase [3]. It is possible that in prostate cancer cells with SPOP mutations, substrates of Cul3-E3 ubiquitin ligase would not be able to go through ubiquitin-dependent degradation, leading to their accumulation and increased activity, which contribute to the pathogenesis of prostate cancer. Identification of the substrates of SPOP could help understand the mechanism of prostate cancer formation and development of a new therapy.

Among the known SPOP substrates reported in literature, SPOP interacts with Daxx, a multifunctional protein regulating transcription, cell cycle and apoptosis [2], SRC-3/AIB1, an oncogenic steroid receptor co-activator-3 [4], Gli of the hedgehog pathway transcriptional activator [5] and DEK [6]. Among these genes, SRC-3 is particularly interesting as a co-activator of transcription activation by androgen receptor (AR), and potentially important in the pathogenesis of prostate cancer [7]. It was reported that SRC-3 protein was overexpressed in 38\% of tumor samples of prostate cancer [8]. Another study showed that SRC-3 expression is required for prostate cancer cell proliferation and survival, and its levels correlated with Prostate Specific Antigen (PSA) [9]. These studies suggest an important role of SRC-3 in prostate cancer formation. A recent article confirmed the hypothesis that mutated SPOP cannot interact with SRC-3 and unable to induce ubiquitin-dependent degradation of SRC-3 [3]. In the current study, a rationale-based approach will be used to target a member of the AR transcriptional complex to suppress the activity of SRC-3 and the androgen receptor complex.

Lestaurtinib has been shown to be a potent inhibitor for PRK-1 [10]. PRK-1 is a serine/threonine kinase of the PKC family and can be inhibited by staurosporin-like members of the PKC family. PRK-1 was shown to play an important role in the androgen receptor (AR) complex [11]. PRK-1 activation results in ligand-dependent superactivation of AR complex even in the presence of androgen receptor antagonist. Considering its proven activity of lestaurtinib in xenograft models [12] and as a targeted therapy for PRK-1, it is possible that lestaurtinib could be effective in prostate cancer, and even more so in a suitable biomarker-defined population. With the fact that lestaurtinib was developed up to a phase 2 randomized study in acute myelocytic leukemia [13], a repurpose application of lestaurtinib in prostate cancer could be a fast way with its established safety and tolerability profile. In this study, we propose a model to explain the pathogenesis of SPOP mutations in prostate cancer and used the model to predict inhibition of SRC3 with lestaurtinib could be a useful therapy for prostate cancer with SPOP mutations.

\section{Materials and Methods}

Materials and cell lines: PC3, 22RV1, DU145 and LNCaP prostate cancer cells were grown in DMEM (Dulbecco's Modified Eagle Medium.) supplemented with glutamine and 10\% fetal bovine serum (all from Gibco, Life Technologies) in an incubator under $37^{\circ} \mathrm{C}$ and $5 \% \mathrm{CO}_{2}$. Lestaurtinib (>99\% pure, molecular weight 439.64) was purchased from LC laboratory and dissolved in DMSO (Sigma-Aldrich).

Cell proliferation assay: MTT assay was used to determine growth suppression of prostate cancer cells by lestaurtinib. Cells were plated in 96-well plateswith 60\% confluence, and incubated with 0.1\% DMSO (control), 10, 30, 100, 300, 1000, 3000 nM lestaurtinib for 48 hours before MTT assay. Each concentration of lestaurtinib was repeated at least 3 times to minimize variation. Statistical analysis of the data was done in paired t-test.

Protein analysis in cell lysates: Protein concentrations were determined by the colorimetric method using BioRad protein analysis reagents. Equal amounts of proteins were analyzed with SDS-PAGE (sodium dodecyl sulfate polyacrylamide gel electrophoresis) using 4\% - 20\% Criterion ${ }^{\mathrm{TM}} \mathrm{TGX}^{\mathrm{TM}}$ Precast Gels. Western blotting of SRC-3 and PRK-1 was conducted with used rabbit polyclonal antibodies (5E11) against SRC-3 (Cell Signaling Technology, Boston, MA) and PRK-1 (07-557) (Upstate/Millpore, Billerica, MA), respectively.

Subcellular Fractionation: Subcellular fractionation was performed to separate nuclei and cytoplasm from cells. PC3, DU145, 22RV1 and LNCaP cells were incubated with $0.1 \%$ DMSO (control) or $1 \mu$ M lestaurtinib for 
6 hours before harvest for subcellular fractionation. Cells were treated with hypotonic buffer, followed by shearing 25 times with $27 \mathrm{G}$ needles in tuberculin syringes. Differential centrifugation was performed at 3000 $\mathrm{rpm}$ at $4^{\circ} \mathrm{C}$ for 10 minutes, and then supernatants were centrifuged at $15,000 \mathrm{rpm}$ at $4^{\circ} \mathrm{C}$ for 10 minutes and the supernatants were used as cytoplasm. The whole cell lysates, nuclear fractions and cytoplasmic fractions were used for Western blotting. Antibodies against tubulin, actin and histone were used for loading and localization controls.

\section{Results}

\subsection{Searching for Other SPOP Substrates}

PubMed search of SPOP-dependent protein degradation revealed that SRC-3, Daxx, DEK and Gli are the known substrates of SPOP [2] [4]-[6]. We searched NCBI database with an attempt to identify other SPOP substrates that are involved in androgen receptor signaling. Structural database in the NCBI revealed that three peptide sequences can interact with SPOP. These include 1) KAASADSTTEGTPAD 2) NTLFPDVSSSTH and 3) DEVTSTTSSS. All three peptide sequences are consistent with the consensus sequence of multiple Ser/Thr (underlined) after an acidic residue (marked in bold) previously reported [14]. We performed a BLAST search using the consensus sequences in order to identify other proteins with the consensus sequence, which might be potential SPOP substrates that were not recognized in the past. First, DVSST and EVTSTT were used for BLAST search, but no human protein was found. Next, DSTT was used but obtained too many hits since it has only 4 residues, and it was hard to make any good conclusion regarding whether they could be good physiological substrates. Another BLAST search with DSTSS resulted in some matches and 4 of the proteins are listed below. Other potential candidates containing sequences consistent with the consensus rule include: 1) AN1-type zinc finger protein 6 isoform c or AWP1 2) linker for activation of T-cells family member 1 isoform c 3) Cullin $-4 \mathrm{~B}$ isoform 14 ) Cullin-7 isoform 2, to name a few.

The results seemed promising since Cullin-4B and Cullin-7 were pulled out from the database, and Cullin-4B had multiple peptides that match the consensus rule. However, a PubMed search for any correlation between prostate cancer and Cullin-4B or Cullin-7 failed to get any reference. Since SPOP can interact with Cullin 3, the chance that it interacts with other members of Cullin family is not impossible. The first hit, AN1-type zinc finger protein 6 has the sequence SALDSTSSS, which is very similar to that of ASADSTT used in structural modeling and fulfill the hydrophobic requirement before aspartic acid. The protein was investigated closely in PubMed to look for any potential correlation with prostate cancer.

\subsection{A Model to Explain the Pathogenesis of SPOP Loss-of-Function Mutations in Prostate Cancer}

AN1-type zinc finger protein 6, also called AWP1, is a zinc finger protein that interacts with tumor necrosis factor receptor-associated factor 2 (TRAF2) [15]. Knockdown of AWP1 protein level increased tumor necrosis factor- $\alpha$ (TNF $\alpha$ )-induced cell death by decreasing NFKB activity [15]. In contrast, increased AWP1 protein in cells could decrease cell death and enhance tumor formation. A different action of AWP1 was found through an interaction with PRK-1, a Protein kinase C family serine/threonine kinase [16]. PRK-1 has been tied to androgen receptor (AR) function in regulation of AR-dependent transcription. Stimulation of PRK-1 results in a liganddependent superactivation of AR [11]. It was showed that PRK-1 promoted the formation of a complex comprised of AR and other co-activators. PRK-1 also binds and stimulates AR activity in the presence of adrenal androgens, which are still present in prostate cancer patients receiving androgen ablation therapy [11]. Based on the above information, it was hypothesized that SPOP mutation could lead to accumulation of AWP1, which may have dual effects in decreasing cell death and enhancing PRK-1 signaling and AR superactivation. Therefore, targeting AWP1 or PRK-1 could be a new approach to develop prostate cancer treatment.

Based on the above information, a model was proposed as shown in Figure 1. In normal or prostate cancer cells that do not have SPOP mutations, SPOP substrates such as Gli, SRC-3 and AWP1 could be degraded through SPOP-dependent protein degradation, which regulates AR activity (left panel, Figure 1). However, in prostate cancer cells with SPOP loss-of-function mutations, SPOP substrates such as SRC-3, Gli, AWP1 will not be regulated by the SPOP-dependent protein degradation and thus accumulated in cells, and enhance AR activity. This situation occurs in the $6 \%-15 \%$ prostate cancer that carries SPOP mutations and this subgroup of prostate 

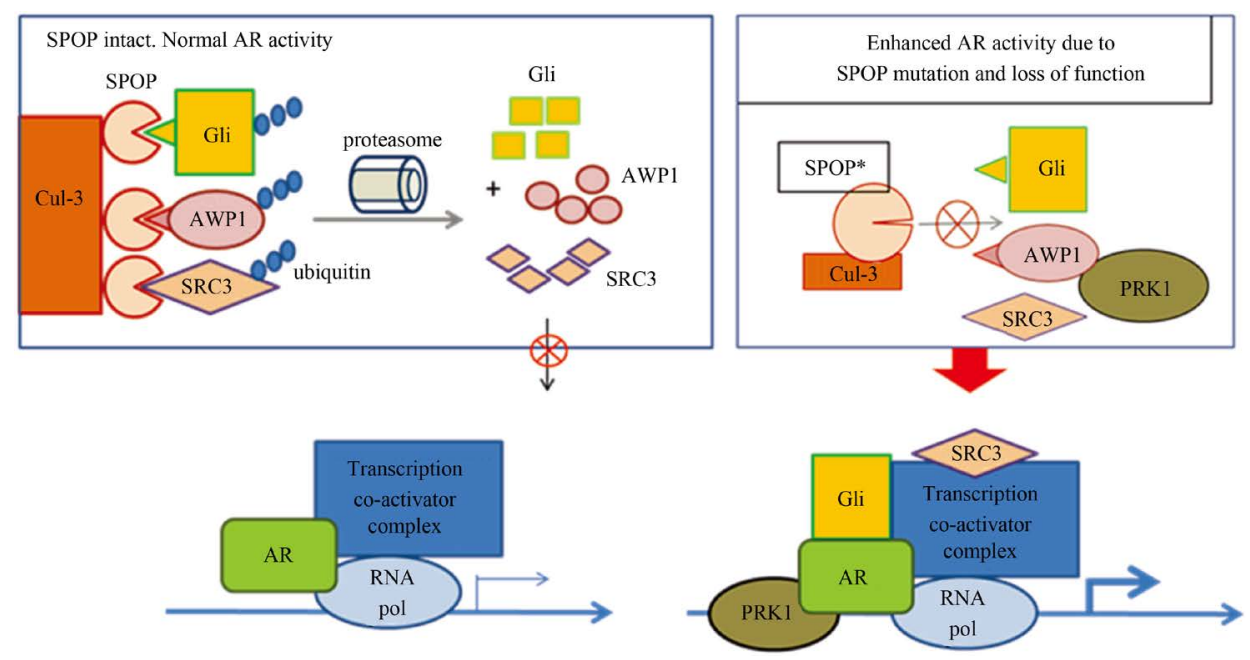

Figure 1. Regulation of AR transcriptional activity in cells with wild-type and mutant SPOP. AR activity is regulated by many proteins, including PRK-1, Gli, SRC-3 transcriptional co-activator. In cells with wild-type SPOP, the levels of the three proteins remain low due to ubiquitin-dependent protein degradation, which subsequently controls AR activity (left panel). In the presence of loss-of-function SPOP mutations, Gli, SRC-3 and AWP1-PRK-1 accumulate to enhance transcriptional activation of AR (right panel) and possibly tumor formation.

cancer may have extraordinary sensitivity to a therapy targeting this complex. This mechanism provides a rationale-based approach to interrupt activation of androgen receptor by blocking the process. Among all players in the pathway, PRK-1, a binding partner of AWP1 and a part of the multiprotein AR transcriptional complex, potentially serves as an easy druggable target due to its nature as a serine/threonine kinase.

\subsection{Blocking PRK-1 by Lestaurtinib Could Be Used to Treat Prostate Cancer with SPOP Mutations}

Next we tested thehypothesis using a compound lestaurtinib, which was reported to be a potent inhibitor of PRK-1and discovered by structural modeling [10]. The same manuscript reported that the $50 \%$ of the maximally inhibitory concentration $\left(\mathrm{IC}_{50}\right.$ ) of lestaurtinib against PRK-1was as low as $8.6 \mathrm{nM}$ [10]. Treatment of LNCaP prostate cancer cells with $5 \mu \mathrm{M}$ lestaurtinib resulted in suppression of several established androgen-inducible targets, including TMPRSS2, Insulin-like growth factor-1 (IGF1), CXC chemokine receptor 4 (CXCR4) and others, but lestaurtinib cannot suppress the androgen-independent genes such as glyceraldehyde-3-phosphate dehydrogenase (GAPDH) [10]. We examined the hypothesis in 4 prostate cancer cell lines to see whether inhibition of PRK-1 by lestaurtinib could be an effective approach against prostate cancer and how it is related to SPOP mutations and other AR transcriptional complex such as SRC-3 and PRK-1.

These prostate cancer cells, PC3, DU145, 22RV1 and LNCaP, were investigated in their sensitivity against lestaurtinib. Cells were treated with lestaurtinib at various concentrations for 48 hours to determine the $\mathrm{IC}_{50}$ using a standard MTT assay. As shown in Figure 2, LNCaP and 22RV1 cells, which are known to be hormonal responsive cell lines, were much more sensitive to lestaurtinib than PC3 and DU145, which are not hormonal responsive, suggesting the notion that the effect of lestaurtinib is mediated by suppression of androgen receptor function.

\subsection{Levels of SRC-3 and PRK-1 Protein Expression in Prostate Cancer Cells}

Using Western blotting, the levels of SRC-3 protein expression were examined in three cell lines before and after lestaurtinib treatment. It was anticipated that cells with higher SRC-3 could have stronger androgen receptor activity. In top panel of Figure 3, the pretreatment levels of SRC-3 were similar in LNCaP, PC3 and DU145 cells. However, after they are treated with lestaurtinib, only the most sensitive LNCaP cells had an obvious decrease of SRC-3. The levels of PRK-1 protein expression were similar before treatment, and had no obvious change after lestaurtinib treatment in all three cells. 


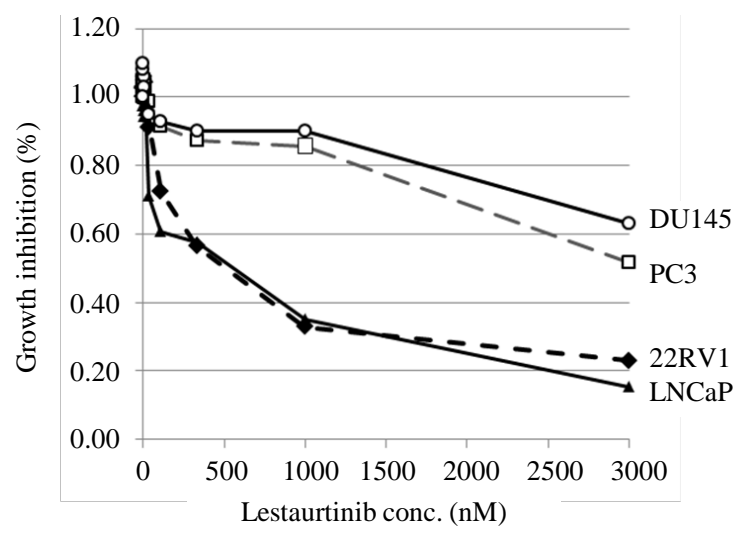

Figure 2. Cell growth suppression by lestaurtinib. Prostate cancer cells PC3, 22RV1, DU145 and LNCaP cells were treated with DMSO (control), 10, 30, 100, 300, 1000, $3000 \mathrm{nM}$ lestaurtinib for 48 hours and cell growth inhibition was determined by MTT assays.
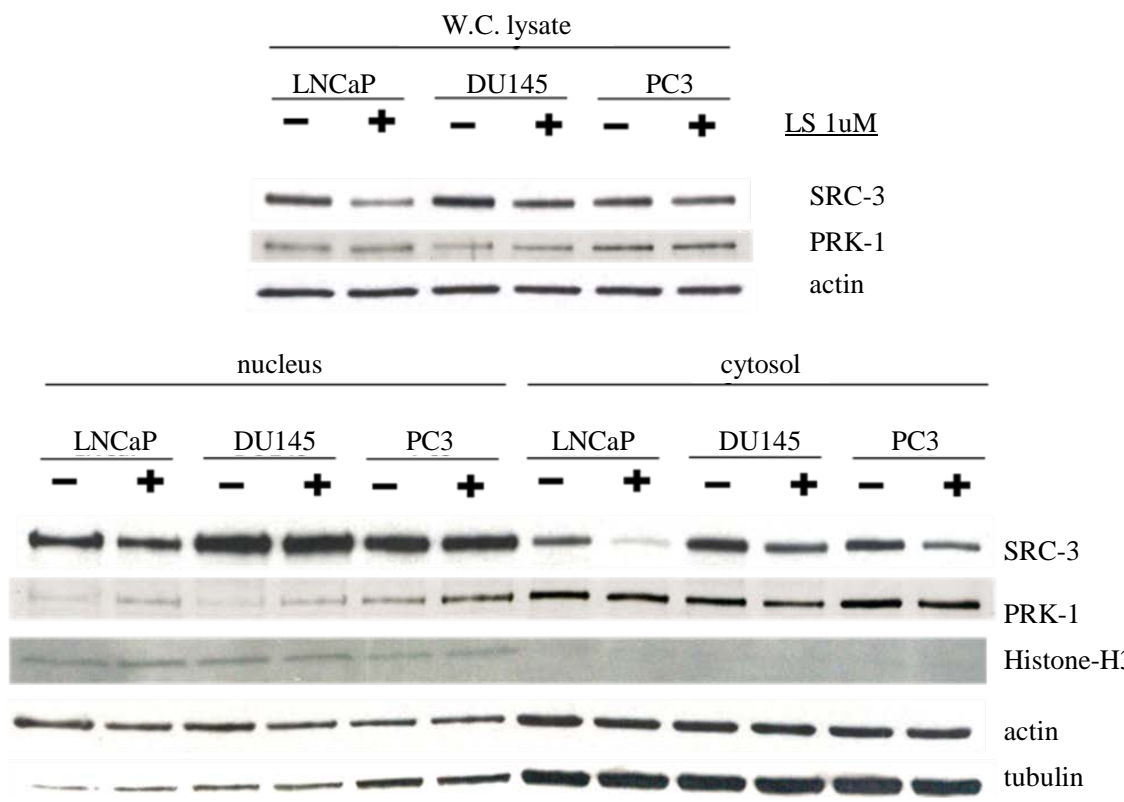

Figure 3. Western blotting of SRC-3 and PRK-1 in prostate cancer cells before and after lestaurtinib treatment. LNCaP, DU145 and PC3 cells were treated with $1 \mu \mathrm{M}$ lestaurtinib for 6 hours and harvested for subcellular fractionation. Whole cell lysates (top panel), nuclear and cytosolic fractions (lower panel) were analyzed with Western blotting using various antibodies as shown in right. Actin, tubulin and Histone-H3 were used as loading and localization controls.

Since SRC-3 and PRK can be shuffling between the cytoplasm and nucleus according to our model in Figure 1, the localization of SRC-3 and PRK was investigated to determine the amount of SRC-3 and PRK-1 in the nucleus and cytoplasm compartments before and after treatment with lestaurtinib. Subcellular fractionation was performed in all three cells before and at 6 hours after treatment with lestaurtinib. The timing of 6 hours was chosen due to the fact that significant morphological changes were observed at this time point (not shown). Each fraction was analyzed with Western blotting with SRC-3 and PRK-1 antibodies, and other markers such as tubulin, histone-H3 and actin were used as loading or localization controls. A decrease of SRC-3 protein was observed mainly in the cytoplasm but not in nucleus after lestaurtinib treatment in all three cell lines. And the decrease was most pronounced in the LNCaP cells, consistent with the fact that this cell line is more sensitive cells to lestaurtinib than the other two (Figure 2). In contrast, PRK-1 protein increased in the nuclear fractions in all 
three cell lines, but no change in cytoplasm after lestaurtinib treatment. The loading control with histone, actin and tubulin confirmed that the cytoplasmic fractions were not contaminated with nucleus.

\section{Discussion}

Recent advance in next generation sequencing of prostate cancer revealed that SPOP is the most commonly mutated gene in $6 \%-15 \%$ of prostate cancer [1]. The localization of mutations at the substrate-binding pocket of SPOP suggests that these mutations could potentially interfere with its ubiquitin ligase activity. We proposed a hypothesis described in Figure 1 that loss-of-function mutations of SPOP lead to accumulation of its physiological substrates, such as SRC-3, which subsequently enhances androgen receptor activity and prostate cancer formation. The hypothesis was tested by searching for an inhibitor that can block a member of the androgen receptor transcriptional complex. PRK-1, a serine/threonine kinase of the PKC family, was chosen as the candidate molecular target in the androgen receptor complex [11], since kinase inhibitors are readily available and easier to develop than blocking protein-protein interaction in the complex. Lestaurtinib was identified in the literature for its potent activity against PRK-1 [10]. The fact that lestaurtinib can suppress androgen-inducible gene expression is another supporting evidence that lestaurtinib can potentially be used in androgen-sensitive prostate cancer. Although previously lestaurtinib failed in a clinical study of androgen-refractory prostate cancer [17], the reason for the failure could be due to selection of a wrong population of prostate cancer. Figure 2 showed the resistance of PC3 and DU145 cells, two androgen-independent prostate cancer cell lines, and supported this possibility. Further evaluation of lestaurtinib in androgen-sensitive prostate cancer, especially for a subpopulation with high androgen receptor activity due to SPOP mutations or high expression of SRC-3, is worth to pursue.

In vitro studies of blocking PRK-1 by lestaurtinib were performed in 3 different cell lines. It was investigated whether the cytotoxic activity of lestaurtinib against prostate cancer cells could be correlated with the protein levels of SRC-3 and presence of SPOP mutations. However, among the three cell lines tested in this project, none of them carries SPOP mutation (data not shown), but this is not surprising as only $6 \%-15 \%$ of prostate cancer was reported to have SPOP mutations. Since SPOP is the key mechanism for regulation of SRC-3 protein level, the lack of mutation is consistent with the finding that the baseline protein levels of SRC-3 are similar in the three tested cell lines (Figure 3, top panel).

However, a difference in SRC-3 protein level was found after cells were treated with lestaurtinib. LNCaP, the most sensitive cells to lestaurtinib and also androgen-sensitive, showed the most prominent decrease of SRC-3 protein after lestaurtinib treatment. In addition, based on the subcellular fractionation studies, the decrease of SRC-3 protein was predominantly in the cytoplasm but not in the nucleus. How can we explain the decrease of SRC-3 protein in the cytoplasm after treatment with lestaurtinib, a serine/threonine kinase inhibitor? Regulation of SRC-3 protein is a complicated process, and phosphorylation of SRC-3 at multiple locations by various kinases has been reported [18]. One kinase that phosphorylates SRC-3 is the atypical PKC family, which could also be inhibited by lestaurtinib due to the similar structures between lestaurtinib and staurosporin, a well-known inhibitor of PKC family members. It was reported that aPKC $\zeta$-induced SRC-3 phosphorylation protects SRC-3 from proteasome-dependent degradation in an ER $\alpha$-dependent manner, leading to an increased estrogen-induced breast cancer cell growth [18]. This mechanism could explain the predominantly decrease of SRC-3 (Figure 3). This decrease of SRC-3 protein level may also contribute to a decrease in androgen receptor activity and enhance the cytotoxic activity of lestaurtinib. Although the nuclear SRC-3 protein levels did not change as dramatically, but it is still possible that blocking PRK-1 activity with lestaurtinib still interferes with SRC-3-induced androgen receptor activity. Animal studies in the past did support the efficacy of lestaurtinib in prostate cancer model [19] [20], in particularly in combination with androgen ablation [20]. Although the clinical toxicity profile of lestaurtinib is tested in acute myelocytic leukemia following standard chemotherapy [13], safety profile in prostate cancer patients remains to be studied. Further investigation of the androgen receptor complex after lestaurtinib treatment would help to solve the problem and move to clinical application.

\section{Conclusion}

This project intended to develop a new approach to treat a subpopulation of prostate cancer with SPOP mutations. This subpopulation of prostate cancer was anticipated to have high androgen receptor activity due to accumulation of SPOP substrates such as SRC-3 and recruitment of PRK-1 in the androgen receptor complex. 
Lestaurtinib was potent in induction of cell death inandrogen-sensitive prostate cancer cells [20] due to its activity in blocking PRK-1 and decreasing SRC-3 protein. Androgen-sensitive prostate cancer, particularly those with confirmed SPOP mutations, could be tested clinically to confirm this hypothesis. Our model suggests that a combination of androgen blockade with lestaurtinib as a two-prong attack could be an effective approach to treat prostate cancer with SPOP mutations.

\section{Acknowledgements}

We thank Drs. David Durrant and Rakesh Kukreja at the Virginia Commonwealth University, Richmond, VA, for the technical support and providing facility for this study.

\section{References}

[1] Barbieri, C.E., Baca, S.C., Lawrence, M.L., et al. (2012) Exome Sequencing Identifies Recurrent SPOP, FOXA1 and MED12 Mutations in Prostate Cancer. Nature Genetics, 44, 685-689. http://dx.doi.org/10.1038/ng.2279

[2] Kwon, J.E., La, M., Oh, K.H., et al. (2006) BTB Domain-Containing Speckle-Type POZ Protein (SPOP) Serves as an Adaptor of Daxx for Ubiquitination by Cul3-Based Ubiquitin Ligase. The Journal of Biological Chemistry, 281, 12664-12672. http://dx.doi.org/10.1074/jbc.M600204200

[3] Geng, C., He, B., Xu, L., Barbieri, C.E., Eedunuri, V.K., et al. (2013) Prostate Cancer-Associated Mutations in Speckle-Type POZ Protein (SPOP) Regulate Steroid Receptor Coactivator 3 Protein Turnover. Proceedings of the National Academy of Sciences of the USA, 110, 6997-7002. http://dx.doi.org/10.1073/pnas.1304502110

[4] Li, C., Ao, J., Fu, J., et al. (2011) Tumor-Suppressor Role for the SPOP Ubiquitin Ligase in Signal-Dependent Proteolysis of the Oncogenic Co-Activator SRC-3/AIB1. Oncogene, 30, 4350-4364. http://dx.doi.org/10.1038/onc.2011.151

[5] Zhang, Q., Zhang, L., Wang, B., et al. (2006) A Hedgehog-Induced BTB Protein Modulates Hedgehog Signaling by Degrading Ci/Gli Transcription Factor. Developmental Cell, 10, 719-729. http://dx.doi.org/10.1016/j.devcel.2006.05.004

[6] Theurillat, J.P., Udeshi, N.D., Errington, W.J., Svinkina, T., Baca, S.C., et al. (2014) Prostate Cancer. Ubiquitylome Analysis Identifies Dysregulation of Effector Substrates in SPOP-Mutant Prostate Cancer. Science, 346, 85-89. http://dx.doi.org/10.1126/science.1250255

[7] Xu, J., Wu, R.C. and O’Malley, B.W. (2009) Normal and Cancer-Related Functions of the p160 Steroid Receptor CoActivator (SRC) Family. Nature Reviews Cancer, 9, 615-630. http://dx.doi.org/10.1038/nrc2695

[8] Gnanapragasam, V.J., Leung, H.Y., Pulimood, A.S., Neal, D.E. and Robson, C.N. (2001) Expression of RAC 3, a Steroid Hormone Receptor Co-Activator in Prostate Cancer. British Journal of Cancer, 85, 1928-1936. http://dx.doi.org/10.1054/bjoc.2001.2179

[9] Zhou, H.J., Yan, J., Luo, W., Ayala, G., Lin, S.H., Erdem, H., Ittmann, M., Tsai, S.Y. and Tsai, M.J. (2005) SRC-3 Is Required for Prostate Cancer Cell Proliferation and Survival. Cancer Research, 65, 7976-7983.

[10] Köhler, J., Erlenkamp, G., Eberlin, A., Rumpf, T., Slynko, I., Metzger, E., Schüle, R., Sippl, W. and Jung, M. (2012) Lestaurtinib Inhibits Histone Phosphorylation and Androgen-Dependent Gene Expression in Prostate Cancer Cells. PLoS One, 7, e34973. http://dx.doi.org/10.1371/journal.pone.0034973

[11] Metzger, E., Müller, J.M., Ferrari, S., Buettner, R. and Schüle, R. (2003) A Novel Inducible Transactivation Domain in the Androgen Receptor: Implications for PRK in Prostate Cancer. The EMBO Journal, 22, 270-280. http://dx.doi.org/10.1093/emboj/cdg023

[12] Weeraratna, A.T., Dalrymple, S.L., Lamb, J.C., Denmeade, S.R., Miknyoczki, S., Dionne, C.A. and Isaacs, J.T. (2001) Pan-Trk Inhibition Decreases Metastasis and Enhances Host Survival in Experimental Models as a Result of Its Selective Induction of Apoptosis of Prostate Cancer Cells. Clinical Cancer Research, 7, 2237-2245.

[13] Levis, M., Ravandi, F., Wang, E.S., Baer, M.R., Perl, A., Coutre, S., Erba, H., Stuart, R.K., et al. (2011) Results from a Randomized Trial of Salvage Chemotherapy Followed by Lestaurtinib for Patients with FLT3 Mutant AML in First Relapse. Blood, 117, 3294-3301. http://dx.doi.org/10.1182/blood-2010-08-301796

[14] Zhang, Q., Shi, Q., Chen, Y., Yue, T., Li, S., Wang, B. and Jiang, J. (2009) Multiple Ser/Thr-Rich Degrons Mediate the Degradation of Ci/Gli by the Cul3-HIB/SPOP E3 Ubiquitin Ligase. Proceedings of the National Academy of Sciences of the United States of America, 106, 21191-21196. http://dx.doi.org/10.1073/pnas.0912008106

[15] Chang, E.J., Ha, J., Kang, S.S., Lee, Z.H. and Kim, H.-H. (2011) AWP1 Binds to Tumor Necrosis Factor ReceptorAssociated Factor 2 (TRAF2) and Is Involved in TRAF2-Mediated Nuclear Factor- $\kappa$ B Signaling. The International Journal of Biochemistry \& Cell Biology, 43, 1612-1620. http://dx.doi.org/10.1016/j.biocel.2011.07.010

[16] Duan, W., Sun, B., Li, T.W., Tan, B.J., Lee, M.K. and Teo, T.S. (2000) Cloning and Characterization of AWP1, a 
Novel Protein That Associates with Serine/Threonine Kinase PRK-1 in Vivo. Gene, 256, 113-121. http://dx.doi.org/10.1016/S0378-1119(00)00365-6

[17] Collins, C., Carducci, M.A., Eisenberger, M.A., Isaacs, J.T., Partin, A.W., Pili, R., Sinibaldi, V.J., Walczak, J.S. and Denmeade, S.R. (2007) Preclinical and Clinical Studies with the Multi-Kinase Inhibitor CEP-701 as Treatment for Prostate Cancer Demonstrate the Inadequacy of PSA Response as a Primary Endpoint. Cancer Biology \& Therapy, 6, 1360-1367. http://dx.doi.org/10.4161/cbt.6.9.4541

[18] Yi, P., Feng, Q., Amazit, L., Lonard, D.M., Tsai, S.Y., Tsai, M.J. and O’Malley, B.W. (2008) Atypical Protein Kinase C Regulates Dual Pathways for Degradation of the Oncogenic Coactivator SRC-3/AIB1. Molecular Cell, 29, 465-476. http://dx.doi.org/10.1016/j.molcel.2007.12.030

[19] Festuccia, C., Muzi, P., Gravina, G.L., Millimaggi, D., Speca, S., Dolo, V., Ricevuto, E., Vicentini, C. and Bologna, M. (2007) Tyrosine Kinase Inhibitor CEP-701 Blocks the NTRK1/NGF Receptor and Limits the Invasive Capability of Prostate Cancer Cells in Vitro. International Journal of Oncology, 30, 193-200. http://dx.doi.org/10.3892/ijo.30.1.193

[20] George, D.J., Dionne, C.A., Jani, J., Angeles, T., Murakata, C., Lamb, J. and Isaacs, J.T. (1999) Sustained in Vivo Regression of Dunning H Rat Prostate Cancers Treated with Combinations of Androgen Ablation and Trk Tyrosine Kinase Inhibitors, CEP-751 (KT-6587) or CEP-701 (KT-5555). Cancer Research, 59, 2395-2401. 